DFTUSA 96/27

\title{
Symplectic Structures and Quantum Mechanics 『
}

\author{
by
}

Giuseppe Marmo ${ }^{1}$ and Gaetano Vilasi ${ }^{2}$

\author{
${ }^{1}$ Dipartimento di Scienze Fisiche, Università di Napoli, \\ Mostrad'Oltremare, Pad.19 - I-80125 Napoli, Italy. (gimarmo@napoli.infn.it) \\ ${ }^{2}$ Dipartimento di Fisica Teorica e smsa, Università di Salerno, \\ Via S. Allende, I-84081 Baronissi (SA), Italy. (vilasi@vaxsa.csied.unisa.it) \\ and \\ Istituto Nazionale di Fisica Nucleare, Sezione di Napoli, Italy.
}

\begin{abstract}
Canonical coordinates for the Schrödinger equation are introduced, making more transparent its Hamiltonian structure. It is shown that the Schrödinger equation, considered as a classical field theory, shares with Liouville completely integrable field theories the existence of a recursion operator which allows for the infinitely many conserved functionals pairwise commuting with respect to the corresponding Poisson bracket.

The approach may provide a good starting point to get a clear interpretation of Quantum Mechanics in the general setting, provided by Stone-von Neumann theorem, of Symplectic Mechanics. It may give new tools to solve in the general case the inverse problem of quantum mechanics whose solution is given up to now only for one-dimensional systems by the Gel'fand-Levitan-Marchenko formula.
\end{abstract}

\section{Introduction.}

In the past few years there has been a renewed interest in completely integrable Hamiltonian systems, specially in connection with the study of integrable quantum field theory, Yang-Baxter algebras and, more recently, quantum groups.

\footnotetext{
${ }^{1}$ Supported in part by the italian Ministero dell' Università e della Ricerca Scientifica e Tecnologica. PACS Nos.:03.20+i,03.65-W.
} 
Loosely speaking, completely integrable Hamiltonian systems are dynamical systems admitting a Hamiltonian description and possessing sufficiently many constants of motion so that they can be integrated by quadratures.

For two-dimensional field theories, a priori criteria of integrability, have been established only by methods more directly related to group theory ${ }^{1,2}$ and to familiar procedures of classical mechanics, looking at such systems as dynamics on (infinitedimensional) phase manifold ${ }^{3,4,5,6,7,8}$

This point of view was also suggested by the occurrence in such models of a peculiar operator, the so called recursion operator ${ }^{9}$, relevant for the effectiveness of the method, which naturally fits in this geometrical setting as a mixed tensor field on the phase manifold M.

In terms of such an operator the classical Liouville theorem on the integrability can be extended also to the infinite dimensional case. The same operator can be used to deal with Burgers equation ${ }^{10}$.

Some years ago it was suggested ${ }^{11}$ the use of complex canonical coordinates in the formulation of a generalized dynamics including classical and quantum mechanics as special cases. In the same spirit a somehow dual viewpoint is proposed: rather than to complexify classical mechanics it is useful to give a formulation of quantum mechanics in terms of realified vector spaces.

By using the Stone-von Neumann theorem a quantum mechanical system is associated with a vector field on some Hilbert space (Schrödinger picture) or a vector field, i.e. a derivation, on the algebra of observables (Heisemberg picture).

In classical mechanics the analog infinitesimal generator of canonical transformations is a vector field on a symplectic manifold (the phase space).

Therefore, if we want to use similar procedures, we need to real off $L_{2}(Q, \mathbf{C})$, the Hilbert space of square integrable complex functions defined on the configuration space $Q$, as a symplectic manifold or, more specifically, as a cotangent bundle. We shall see that it can be considered as $T^{*}\left(L_{2}(Q, \mathbf{R})\right), \quad L_{2}(Q, \mathbf{R})$ denoting the Hilbert space of square integrable real functions defined on $Q$.

This approach is different from previous ones ${ }^{12}$ also dealing with the integrability of quantum mechanical system in the Heisemberg and Schrödinger picture. 
In order to make more transparent the geometrical and the physical content of the paper difficult technical aspects, which are however important in the context of infinite dimensional manifold, as, for instance, the distinction ${ }^{13}$ between weakly and strongly not degenerate bilinear forms, or the inverse of a Schrödinger operator and so on, will not be addressed. We shall limit ourselves to observe that no serious difficulties arise working on an infinite dimensional manifold whose local model is a Banach space, as in that case the implicit function theorem still holds true.

\section{Complete Integrability and Recursion Operators}

Complete integrability of Hamiltonian systems with finitely many degrees of freedom is exhaustively characterized by the Liouville-Arnold theorem ${ }^{14,15}$. An alternative characterization which may apply also to systems with infinitely many degrees of freedom can be given as follows. Let $\mathrm{M}$ denote a smooth differentiable manifold, $\mathcal{X}(M)$ and $\Lambda(M)$ vector and covector fields on $\mathrm{M}$. With any $(1,1)$ tensor field $T$ on $M$, two endomorphisms

$$
\hat{T}: \mathcal{X}(M) \rightarrow \mathcal{X}(M) \quad \text { and } \quad \check{T}: \Lambda(M) \rightarrow \Lambda(M)
$$

are associated:

$$
T(a, X)=<\alpha, \hat{T} X>=<\check{T} \alpha, X>,
$$

with $X$ and $\alpha$ belonging to $\mathcal{X}(M)$ and $\Lambda(M)$ respectively. The Nijenhuis tensor ${ }^{16}$, or torsion, of $T$ is the $(1,2)$ tensor field defined by:

$$
N_{T}(\alpha, X, Y)=<\alpha, H_{T}(X, Y)>
$$

with the vector field $H_{T}(X, Y)$ given by:

$$
H_{T}(X, Y)=\left[\widehat{\mathcal{L}_{\hat{T} X} T}-\hat{T} \widehat{\mathcal{L}_{X} T}\right] Y
$$

$\mathcal{L}_{X}$ denoting the Lie's derivative with respect to $X$.

\section{Integrability Criterium円}

A dynamical vector field $\Delta$ which admits an invariant mixed tensor field $T$, with vanishing Nijenhuis tensor $N_{T}$ and bidimensional eigenspaces, completely separates

\footnotetext{
${ }^{1}$ The vector field $\Delta$ is not supposed to be Hamiltonian. Its Hamiltonian structure is generated by the hypothesis of the bidimensionality of the eigenspaces of $\mathrm{T}$ and $d \lambda \neq 0$.
} 
in 1-degree of freedom dynamics. The ones associated with those degrees of freedom whose corresponding eigenvalues $\lambda$ are not stationary, are integrable and Hamiltonian ${ }^{4}$.

An idea of the proof is given observing that the bidimensionality of eigenspaces of $T$ and the condition $N_{T}=0$ imply the following form for $T$

$$
T=\sum_{i} \lambda_{i}\left(\frac{\delta}{\delta \lambda^{i}} \otimes \delta \lambda^{i}+\frac{\delta}{\delta \phi^{i}} \otimes \delta \phi^{i}+\frac{\delta}{\delta \phi^{i}} \otimes \delta \lambda^{i}\right)+\sum_{\ell=1}^{2} \int_{0}^{k} d k \quad k \frac{\delta}{\delta \psi_{k}^{\ell}} \otimes \delta \psi^{\ell}(k)
$$

The invariance of $T\left(\mathcal{L}_{\Delta} T=0\right)$ implies for $\Delta$ the form

$$
\Delta=\sum_{i=1}^{n} \Delta^{i}\left(\lambda^{i}\right) \frac{\delta}{\delta \phi^{i}}+\sum_{\ell=1}^{2} \int d k \Delta^{\ell}(k)\left(\psi^{1}(k), \psi^{2}(k)\right) \frac{\delta}{\delta \psi^{\ell}(k)}
$$

whose associated equations are:

$$
\begin{aligned}
& \dot{\psi}^{1}(k)=\Delta^{1, k}\left(\psi^{1,(k)}, \psi^{2,(k)}\right) \\
& \dot{\psi}^{2,(k)}=\Delta^{2, k}\left(\psi^{1,(k)}, \psi^{2,(k)}\right) \\
& \dot{\phi}^{i}=\Delta^{i}\left(\lambda^{i}\right) \\
& \dot{\lambda}^{i}=0
\end{aligned}
$$

For the discrete part of the spectrum of $T$ a symplectic form $\omega_{0}$ can be defined $\omega_{0}=\sum_{i} f_{i}\left(\lambda^{i}\right) \delta \lambda^{i} \wedge \delta \phi^{i}$ with respect to which the dynamics is a Hamiltonian one.

In next section the mentioned geometrical structures will be exhibited for the Schrödinger equation.

\section{Canonical Coordinates for the Schrödinger equation}

Although in an infinite dimensional symplectic manifold a Darboux's chart, a priori does not exist, for the Schrödinger equation:

$$
i \hbar \frac{\partial \psi}{\partial t}=-\frac{\hbar^{2}}{2 m} \triangle \psi+U(\mathbf{r}) \psi
$$

natural canonical coordinates $p$ and $q$ can be introduced.

We introduce the real and the imaginary part of the wave function $\psi$ :

$$
\left\{\begin{array}{l}
p(\mathbf{r}, t)=\operatorname{Im} \psi(\mathbf{r}, t) \\
q(\mathbf{r}, t)=\operatorname{Re} \psi(\mathbf{r}, t)
\end{array}\right.
$$


and in this way $L_{2}(Q, \mathbf{C})$ is considered as the cotangent bundle of $L_{2}(Q, \mathbf{R})$.

In these new coordinates, equation (4) takes the form:

$$
\frac{d}{d t}\left(\begin{array}{l}
p \\
q
\end{array}\right)=\frac{1}{\hbar}\left(\begin{array}{cc}
0 & -1 \\
1 & 0
\end{array}\right)\left(\begin{array}{c}
\frac{\delta H_{1}}{\delta p} \\
\frac{\delta H_{1}}{\delta q}
\end{array}\right)
$$

where $H_{1}$ is defined by:

$$
H_{1}[q, p]:=\frac{1}{2} \int d \mathbf{r}\left\{\frac{\hbar^{2}}{m}\left[(\nabla p)^{2}+(\nabla q)^{2}\right]+U(\mathbf{r})\left(p^{2}+q^{2}\right)\right\}
$$

and $\frac{\delta H}{\delta q}, \frac{\delta H}{\delta p}$ denote the components of the gradient of $H[q, p]$ with respect to the real $L_{2}$ scalar product.

Our system is then a Hamiltonian dynamical system with respect to the Poisson bracket defined for any two functionals $F[q, p]$ and $G[q, p]$ by:

$$
\Lambda_{1}(\delta F, \delta G):=\{F, G\}_{1}:=\frac{1}{\hbar} \int d \mathbf{r}\left(\frac{\delta F}{\delta q} \cdot \frac{\delta G}{\delta p}-\frac{\delta F}{\delta p} \cdot \frac{\delta G}{\delta q}\right)
$$

What is less known is that the previous one is not the only possible Hamiltonian structure . As matter of fact the Schrödinger equation can also be written as:

$$
\frac{d}{d t}\left(\begin{array}{l}
p \\
q
\end{array}\right)=\frac{1}{\hbar}\left(\begin{array}{cc}
0 & -\mathcal{H} \\
\mathcal{H} & 0
\end{array}\right)\left(\begin{array}{c}
\frac{\delta H_{0}}{\delta p} \\
\frac{\delta H_{0}}{\delta q}
\end{array}\right)
$$

where $H_{0}$ is defined by:

$$
H_{0}[q, p]:=\frac{1}{2} \int d \mathbf{r}\left(p^{2}+q^{2}\right)
$$

and $\mathcal{H}$ is the Schrödinger operator:

$$
\mathcal{H}:=-\frac{\hbar^{2}}{2 m} \triangle+U(\mathbf{r})
$$

It is then again a Hamiltonian dynamical systems with a new Poisson bracket of any two functionals $F[q, p]$ and $G[q, p]$ given by:

$$
\Lambda_{0}(\delta F, \delta G):=\{F, G\}_{0}:=\int d \mathbf{r}\left(\frac{\delta F}{\delta q} \cdot \mathcal{H} \frac{\delta G}{\delta p}-\frac{\delta F}{\delta p} \cdot \mathcal{H} \frac{\delta G}{\delta q}\right)
$$

So, with the same vector field, we have two choices: 
- A phase manifold with a universal symplectic structure:

$$
\omega_{1}:=\hbar \int d \mathbf{r}(\delta p \wedge \delta q)
$$

and a Hamitonian functional depending on the classical potential.

- A phase manifold with a symplectic structure determined by the classical potential

$$
\omega_{0}:=\hbar \int d \mathbf{r}\left(\mathcal{H}^{-1} \delta p \wedge \delta q\right)
$$

and the universal Hamiltonian functional representing the quantum probability.

The two brackets satisfy the Jacobi Identity, as the associated 2-forms are closed for they do not depend on the point $(\psi \equiv(p, q))$ of the phase space.

We have then the relation:

$$
\frac{\delta H_{1}}{\delta u}=\check{T} \frac{\delta H_{0}}{\delta u}
$$

where:

$$
\check{T}:=\Lambda_{1}^{-1} \circ \Lambda_{0}=\left(\begin{array}{cc}
\mathcal{H} & 0 \\
0 & \mathcal{H}
\end{array}\right)
$$

and

$$
\frac{\delta H}{\delta u}=\left(\begin{array}{c}
\frac{\delta H}{\delta q} \\
\frac{\delta H}{\delta p}
\end{array}\right)
$$

As the tensor field $\mathrm{T}$ does not depend on the point $(\psi \equiv(p, q))$ of the phase space, its torsion is identically zero, so that the relation (14) can be iterated to:

$$
\frac{\delta H_{n}}{\delta u}=\check{T}^{n} \frac{\delta H_{0}}{\delta u}
$$

It turns out that the Schrödinger equation admits infinitely many conserved functionals defined by:

$$
H_{n}[q, p]:=\frac{1}{2} \int d \mathbf{r}\left(p \mathcal{H}^{n} p+q \mathcal{H}^{n} q\right) \equiv \int d \mathbf{r}\left(\bar{\psi} \mathcal{H}^{n} \psi\right)
$$

They are all in involution with respect to the previous Poisson brackets:

$$
\left\{H_{n}, H_{m}\right\}_{0}=\left\{H_{n}, H_{m}\right\}_{1}=0
$$


This situation generalizes the one for finite dimensional Hamiltonian systems ${ }^{4}$. It is worth to stress that for smooth potentials $U(x)$ in one space dimension, the eigenvalues of the Schrödinger operator $\mathcal{H}$ are not degenerate and so the eigenvalues of $T$ are double degenerate.

\subsection{The eikonal transformation}

The transformation:

$$
\left\{\begin{array}{l}
p(\mathbf{r}, t)=A(\mathbf{r}, t) \sin S(\mathbf{r}, t) \hbar^{-1} \\
q(\mathbf{r}, t)=A(\mathbf{r}, t) \cos S(\mathbf{r}, t) \hbar^{-1}
\end{array}\right.
$$

is a canonical transformation between the $(p, q)$ coordinates and $\left(\pi=S(2 \hbar)^{-1} J, \chi=\right.$ $\left.A^{2}\right)$, as:

$$
\delta p \wedge \delta q=\delta\left(\frac{S}{2 \hbar}\right) \wedge \delta A^{2}
$$

The Hamiltonian $H_{1}$ becomes:

$$
K_{1}[\chi, \pi]=\int d \mathbf{r}\left\{\frac{\hbar^{2}}{2 m}\left(\frac{(\nabla \chi)^{2}}{4 \chi}+4 \chi(\nabla \pi)^{2}\right)+U \chi\right\}
$$

and Hamilton's equations:

$$
\left\{\begin{array}{l}
\frac{\partial \pi}{\partial t}=-\frac{1}{\hbar} \frac{\delta K_{1}}{\delta \chi} \\
\frac{\partial \chi}{\partial t}=\frac{1}{\hbar} \frac{\delta K_{1}}{\delta \pi}
\end{array}\right.
$$

give:

$$
\left\{\begin{array}{l}
\frac{\partial \pi}{\partial t}=\frac{\hbar}{2 m} \frac{\Delta(\sqrt{\chi})}{\sqrt{\chi}}-\frac{\hbar}{m}(\nabla \pi)^{2}-U \hbar^{-1} \\
\frac{\partial \chi}{\partial t}=-\frac{2 \hbar}{m} \operatorname{div}(\chi \nabla \pi)
\end{array}\right.
$$

where $P=\chi$ and $\mathbf{J}=\hbar \chi \frac{\nabla S}{m}$ represent the probability density and the current density respectively.

This transformation being nonlinear will transform previous biHamiltonian descriptions into a mutually compatible pair of nonlinear type. They are of $C$-type as introduced by Calogero ${ }^{17}$. 


\subsection{The quantum Lagrangians}

Having considered equations of motion for a quantum system as equations for the integral curves of a vector field on a cotangent bundle, it is a natural question to ask if this vector field may be associated with a Lagrangian vector field on a tangent bundle.

This question for a Lagrangian Schrödinger Equation can be answered as follows: From equation (5) one gets Hamilton's equations:

$$
\left\{\begin{array}{l}
\frac{\partial p}{\partial t}=-\frac{1}{\hbar} \mathcal{H} q \\
\frac{\partial q}{\partial t}=\frac{1}{\hbar} \mathcal{H} p
\end{array}\right.
$$

from which we derive the second order equation :

$$
\frac{\partial^{2} q}{\partial t^{2}}=-\frac{1}{\hbar^{2}} \mathcal{H}^{2} q
$$

The latter is the Euler-Lagrange equation associated with the Lagrangian functional:

$$
L_{1}[q]=\frac{1}{2} \int d \mathbf{r} d t\left(q_{t}^{2}-\frac{1}{\hbar^{2}} q \mathcal{H}^{2} q\right)
$$

Of course the Legendre transformation

$$
\pi=\frac{\delta L_{1}}{\delta q_{t}}
$$

does not give the Hamilton's equation (25) but the related one:

$$
\left\{\begin{array}{l}
\frac{\partial \pi}{\partial t}=-\frac{1}{\hbar^{2}} \mathcal{H}^{2} q \\
\frac{\partial q}{\partial t}=\pi
\end{array}\right.
$$

Equations (25) follows straightforward from the Lagrangian $L_{0}$ given by:

$$
L_{0}[q]=\frac{1}{2} \int d \mathbf{r} d t\left(q_{t} \mathcal{H}^{-1} q_{t}-\frac{1}{\hbar^{2}} q \mathcal{H} q\right)
$$

Of course $L_{0}$ is the Lagrangian which gives rise to the $\omega_{0}$ symplectic form and that:

$$
\frac{\delta L_{1}}{\delta q}=\mathcal{H} \frac{\delta L_{0}}{\delta q} ; \quad \frac{\delta L_{1}}{\delta q_{t}}=\mathcal{H} \frac{\delta L_{0}}{\delta q_{t}}
$$

or equivalently: 


$$
\frac{\delta L_{1}}{\delta v}=\check{T} \frac{\delta L_{0}}{\delta v}
$$

where

$$
\frac{\delta L}{\delta v}:=\left(\begin{array}{l}
\frac{\delta L}{\delta q} \\
\frac{\delta L}{\delta q_{t}}
\end{array}\right)
$$

It is also clear that, as in the case of the Hamiltonian functionals, relation (32) can be iterated to give altenative Lagrangian descriptions.

\section{Conclusions}

It has been shown as the Schrödinger equation, considered as a vector field on an infinite dimensional vector space, admits more than one Hamiltonian formulation. Really it admits infinitely many alternative Hamiltonian descriptions in terms of

$$
H_{n}[q, p]:=\frac{1}{2} \int d \mathbf{r}\left(p \mathcal{H}^{n} p+q \mathcal{H}^{n} q\right) \equiv \int d \mathbf{r}\left(\bar{\psi} \mathcal{H}^{n} \psi\right)
$$

and

$$
\omega_{n}:=\hbar \int d \mathbf{r}\left(\mathcal{H}^{n-1} \delta p \wedge \delta q\right)
$$

providing us with the same vector field:

$$
\Delta:=\frac{1}{\hbar} \int d \mathbf{r}\left(\mathcal{H} p \frac{\delta}{\delta q}-\mathcal{H} q \frac{\delta}{\delta p}\right)
$$

defined by:

$$
i_{\Delta} \omega_{n}:=-\delta H_{n}
$$

These are associated with the Lagrangians

$$
L_{n}[q]=\frac{1}{2} \int d \mathbf{r} d t\left(q_{t} \mathcal{H}^{n-1} q_{t}-\frac{1}{\hbar^{2}} q \mathcal{H}^{n+1} q\right)
$$

whose gradients are generated by the tensor field $T$.

Even thought our construction is a formal one, it is understood that the construction applies to any bounded, invertible operator $\mathcal{H}$.

Finally, it is worth to stress that the Schrödinger equation, in spite of its linearity, shows that the class of completely integrable field theories in higher dimensional 
spaces is not empty. Moreover, previous analysis appears to be interesting also in the formulation of variational principles ${ }^{18}$ for stochastic mechanics.

\section{Acknowledgement.}

The authors wish to thank prof. M.Rasetti for his encouragement and comments. One of them (G.V.) is in debt with prof. F.Guerra who stressed the relevance of the approach from the viewpoint of Stochastic Mechanics. 


\section{References}

1. R.Schmid, Infinite Dimensional Hamiltonian Systems (Bibliopolis Naples 1987) and references therein.

2. JL.D.Faddeev and L.A.Takhtajan, Hamiltonian Methods in the Theory of Solitons, (Berlin, Springer 1987), and references therein.

3. G. Vilasi, Phys. Lett. B 94 (1980)195

G. Marmo, A Geometrical Characterization of Completely Integrable Systems in Geometry and Physics (Modugno ed.) (Pitagora, Florence 1982)257.

S. De Filippo, G. Vilasi, Geometrical Methods for Infinite Dimensional Dynamical Systems, Proceedings Second World Conference on Mathematics, (Las Palmas, Spain 1982)236.

S. De Filippo, G. Marmo, G. Vilasi, Phys. Lett. B 117 (1982)418.

G. Marmo, Nijenhuis Operators in Classical Dynamics, Proceedings of Seminar on Group Theoretical Methods in Physics, USSR (1985).

P. Di Stasio and G.Vilasi, Lett. in Math. Phys. 11(1986)299

G. Vilasi, Phys. Lett. B 174 (1986)203

G. Marmo, G. Vilasi, Phys. Lett. B 277 (1992)137.

4. S. De Filippo, G. Marmo, M. Salerno, G. Vilasi, On the Phase Manifold Geometry of Integrable Nonlinear Field Theories.IFUSA n.2 (1982)

S. De Filippo, G. Marmo, M. Salerno, G. Vilasi, Il N. Cimento 83 B, 2 (1984)97.

S. De Filippo, M. Salerno, G. Vilasi, Lett. Math. Phys. 9 (1985) 85.

G.Landi, G.Marmo and G.Vilasi, J.Math. Phys. 35(1994)808

5. I.M. Gel' fand and I. Ya Dorfman, Funct. Anal. 14, 3 (1980)71.

6. F.Magri, J.Math. Phys. 18, (1978)1156; Lect. Notes in Math. 120 (Berlin, Springer 1980)

7. B. Fuchsteiner, Progr. of Theor. Phys. 68, 4 (1982)1082. 
8. V.E.Zakharov, B.G.Konopolchenko, Comm.Math.Phys. 94 (1984)483

Y.Kosmann-Schwarzbach, Geomètrie des systems bihamiltonian, Pub.IRMA, 2,1 Lille(1986)

9. P.D.Lax, Comm.Pure Appl. Math. 21 (1968)467;28 (1975)141; Siam Rev., 18(1976)351

10. S.De Filippo, G.Marmo, M.Salerno, G.Vilasi, Lettere a Il N.Cimento 37, 3 (1983)105.

11. F. Strocchi, Rev.Mod.Phys. (1966)

12. B.A. Kupershmidt, Phys. Lett. A 109 (1985)136.

A.M. Bloch,Phys. Lett. A 116 (1986)353.

R.Cirelli, L.Pizzocchero, On the integrability of quantum mechanics as an infinite dimensional Hamiltonian system, Nonlinearity 3 (1990)

G.Cassinelli, E.De Vito, A.Levrero, The dynamical evolution of quantum systems in the adiabatic approximation, Preprint Università di Genova (1996)

13. R. Chernoff and J.Marsden, Lect. Notes in Math.425 (Berlin, Springer 1974)

14. J. Liouville, Acta Math. 20 (1897)239.

H. Poincarè, Acta Math. 13 (1899)1.

15. V.I.Arnold, Les mèthodes mathèmatiques de la Mècanique Classique (Mir, Moscou 1976)

R.Abraham and J.E.Marsden, Foundations of Mechanics (Benjamin, Massachussets 1978)

16. A.Frolicher and A.Nijenhuis, Indag. Math., 23,(1956)338

A.Nijenhuis, Indag. Math., 49,(1987)2

17. F.Calogero, Physica 18D,(1986)280

18. F.Guerra and L.Morato, Phys.Rev.D27(1983)1774

F.Guerra and R.Marra, Phys.Rev.D28 (1983)1916; D29 (1984)1647 